\title{
Antiferromagnetic Order in the Hubbard Model: Spin-Charge Rotating Reference Frame Approach
}

\author{
T.A. Zaleski And T.K. KopeĆ \\ Institute of Low Temperature and Structure Research \\ Polish Academy of Sciences \\ P.O.B. 1410, 50-950 Wrocław 2, Poland
}

\begin{abstract}
We study the antiferromagnetic phase of three-dimensional Hubbard model with nearest neighbors hopping on a bipartite cubic lattice. We use the quantum $\mathrm{SU}(2) \times \mathrm{U}(1)$ rotor approach that yields a fully self-consistent treatment of the antiferromagnetic state that respects the symmetry properties of the model and satisfies the Mermin-Wagner theorem. As our theory describes the evolution from a Slater $(U \ll t)$ to a Mott-Heisenberg $(U \gg t)$ antiferromagnet, we present the phase diagram of the antiferromagnetic Hubbard model as a function of the crossover parameter $U / t$.
\end{abstract}

PACS numbers: 71.10.Fd, 75.10.Jm

\section{Introduction}

In the realm of strongly correlated electrons, a key question concerns the emergence of low energy scales, much smaller than the bare Coulomb interactions between the electrons, which govern the existence and the competition of different phases. Furthermore, the issue of magnetic ordering in those systems is profoundly complex due to the requirement of self-consistency by incorporating spin and charge fluctuations, while maintaining the essential spin-rotation symmetry. In the present paper, we propose a theoretical approach, which provides a unified view of three-dimensional (3D), half-filled Hubbard model for any value of the Coulomb repulsion $U$, which is able to handle the evolution from the Slater to the Mott-Heisenberg antiferromagnet that captures correctly both the spin and charge degrees of freedom. It is achieved by implementing the charge-U(1) and spin-SU(2) rotationally invariant handling of the Hubbard model. In this scheme the charge and spin excitations emerge in terms of a $\mathrm{U}(1)$ phase and variable spin quantization axis: the effective field theory for the strongly correlated problem is thus characterized by the $\mathrm{U}(2)=\mathrm{U}(1) \times \mathrm{SU}(2)$ group, where the gauge potential in 
$\mathrm{U}(1)$ describes the evolution of a particle scalar characteristic, which is naturally associated with an electric charge, while the gauge potential in $\mathrm{SU}(2)$ describes the nontrivial dynamics associated with the evolution of the vector internal characteristic of a particle such as spin.

\section{The model}

We start with the purely fermionic Hubbard Hamiltonian $\mathcal{H} \equiv \mathcal{H}_{t}+\mathcal{H}_{U}$ :

$$
\mathcal{H}=-t \sum_{\left\langle\boldsymbol{r} \boldsymbol{r}^{\prime}\right\rangle, \alpha}\left[c_{\alpha}^{\dagger}(\boldsymbol{r}) c_{\alpha}\left(\boldsymbol{r}^{\prime}\right)+\text { h.c. }\right]+U \sum_{\boldsymbol{r}} n_{\uparrow}(\boldsymbol{r}) n_{\downarrow}(\boldsymbol{r}),
$$

where $\left\langle\boldsymbol{r}, \boldsymbol{r}^{\prime}\right\rangle$ run over the nearest-neighbor (n.n.) sites, $U$ stands for the Coulomb repulsion, $t$ is the hopping amplitude, and $c_{\alpha}^{\dagger}(\boldsymbol{r})$ is the electron creation operator with spin $\alpha=\uparrow, \downarrow$. The average number of electrons is controlled via an additional term in Eq. (1), $\mathcal{H} \rightarrow \mathcal{H}-\mu \sum_{\boldsymbol{r}} n(\boldsymbol{r})$ with $\mu$ being the chemical potential and $n(\boldsymbol{r})=n_{\uparrow}(\boldsymbol{r})+n_{\downarrow}(\boldsymbol{r})$ the fermionic number operator. Introducing the Grassmann fields, $c_{\alpha}(\boldsymbol{r} \tau)$ depending on the "imaginary time" $0 \leq \tau \leq \beta \equiv 1 / k_{\mathrm{B}} T$ (with $T$ being the temperature) that satisfy the anti-periodic condition $c_{\alpha}(\boldsymbol{r} \tau)=-c_{\alpha}(\boldsymbol{r} \tau+\beta)$, we are able to write the path integral for the statistical sum $\mathcal{Z}=\int[\mathcal{D} \bar{c} \mathcal{D} c] \mathrm{e}^{-\mathcal{S}[\bar{c}, c]}$ with the fermionic action

$$
\mathcal{S}[\bar{c}, c]=\mathcal{S}_{\mathrm{B}}[\bar{c}, c]+\int_{0}^{\beta} \mathrm{d} \tau \mathcal{H}[\bar{c}, c]
$$

that contains the fermionic Berry term [1]:

$$
\mathcal{S}_{\mathrm{B}}[\bar{c}, c]=\sum_{\boldsymbol{r} \alpha} \int_{0}^{\beta} \mathrm{d} \tau \bar{c}_{\alpha}(\boldsymbol{r} \tau) \partial_{\tau} c_{\alpha}(\boldsymbol{r} \tau)
$$

\section{3. $\mathrm{SU}(2) \times \mathrm{U}(1)$ action}

The interaction term in the Hubbard Hamiltonian can be decoupled via a Hubbard-Stratonovitch (HS) transformation by introducing auxiliary fields for the spin and charge fluctuations. Since such a procedure usually leads to a loss of the spin rotational invariance, it is crucial to construct a formulation of the theory which naturally preserves the existing symmetry present in the Hubbard Hamiltonian. To this end, the density-density product in Eq. (1) is written, following Ref. [2], in a spin-rotational invariant way

$$
\mathcal{H}_{U}=U \sum_{\boldsymbol{r}}\left\{\frac{1}{4} n^{2}(\boldsymbol{r} \tau)-[\boldsymbol{\Omega}(\boldsymbol{r} \tau) \cdot \boldsymbol{S}(\boldsymbol{r} \tau)]^{2}\right\},
$$

where $S^{a}(\boldsymbol{r} \tau)=\frac{1}{2} \sum_{\alpha \alpha^{\prime}} c_{\alpha}^{\dagger}(\boldsymbol{r} \tau) \hat{\sigma}_{\alpha \alpha^{\prime}}^{a} c_{\alpha^{\prime}}(\boldsymbol{r} \tau)$ denotes the vector spin operator $(a=$ $x, y, z)$ and $\hat{\sigma}^{a}$ are the Pauli matrices. The unit vector

$$
\boldsymbol{\Omega}(\boldsymbol{r} \tau)=[\sin \vartheta(\boldsymbol{r} \tau) \cos \varphi(\boldsymbol{r} \tau), \sin \vartheta(\boldsymbol{r} \tau) \sin \varphi(\boldsymbol{r} \tau), \cos \vartheta(\boldsymbol{r} \tau)]
$$

written in terms of polar angles labels varying in space-time spin quantization axis, which, in order to maintain spin rotational invariance, should be considered to be a priori arbitrary. Furthermore, the integration in the partition function should 
run over all possible directions of $\boldsymbol{\Omega}(\boldsymbol{r} \tau)$. By decoupling spin and charge density terms in Eq. (4) using auxiliary fields $\varrho(\boldsymbol{r} \tau)$ and $\mathrm{i} V(\boldsymbol{r} \tau)$, respectively, we write down the partition function in the form

$$
\mathcal{Z}=\int[\mathcal{D} \boldsymbol{\Omega}] \int[\mathcal{D} V \mathcal{D} \varrho] \int[\mathcal{D} \bar{c} \mathcal{D} c] e^{-\mathcal{S}[\boldsymbol{\Omega}, V, \varrho, \bar{c}, c]},
$$

where $[\mathcal{D} \boldsymbol{\Omega}] \equiv \prod_{\boldsymbol{r} \tau_{k}} \frac{\sin \vartheta\left(\boldsymbol{r} \tau_{k}\right) \mathrm{d} \vartheta\left(\boldsymbol{r} \tau_{k}\right) \mathrm{d} \varphi\left(\boldsymbol{r} \tau_{k}\right)}{4 \pi}$ is the spin-angular integration measure. This leads us to the effective action

$$
\begin{gathered}
\mathcal{S}[\boldsymbol{\Omega}, V, \varrho, \bar{c}, c]=\sum_{\boldsymbol{r}} \int_{0}^{\beta} \mathrm{d} \tau\left[\frac{\varrho^{2}(\boldsymbol{r} \tau)}{U}+\frac{V^{2}(\boldsymbol{r} \tau)}{U}+\mathrm{i} V(\boldsymbol{r} \tau) n(\boldsymbol{r} \tau)\right. \\
+2 \varrho(\boldsymbol{r} \tau) \boldsymbol{\Omega}(\boldsymbol{r} \tau) \cdot \boldsymbol{S}(\boldsymbol{r} \tau)]+\mathcal{S}_{\mathrm{B}}[\bar{c}, c]+\int_{0}^{\beta} \mathrm{d} \tau \mathcal{H}_{t}[\bar{c}, c] .
\end{gathered}
$$

A systematic way of decomposing the fluctuating fields contained in the action in Eq. (7) enables us to obtain a low energy effective theory.

\section{AF long-range order parameter}

A characteristic property of strongly correlated systems is the existence of local moments, which are usually incorrectly described by weak-coupling theories. With electron correlation effects becoming stronger, spin fluctuations have to be considered carefully. The Hartree-Fock transition temperature has a meaning of a temperature below which the amplitude $\Delta_{\mathrm{c}}$ of the antiferromagnetic $(\mathrm{AF})$ order parameter takes a well-defined value $\left(\Delta_{\mathrm{c}}=U\left\langle S^{z}(\boldsymbol{r} \tau)\right\rangle\right.$ sets the magnitude for the Mott-charge gap). This is also interpreted as the appearance of local moments. However, the existence of AF long-range order requires not only a nonzero value of $\Delta_{\mathrm{c}}$, but also ordered angular degrees of freedom $\boldsymbol{\Omega}(\boldsymbol{r} \tau)$, whose low-lying excitations are in the form of spin waves. In the $\mathrm{CP}^{1}$ representation (where the Néel field is represented by two Schwinger bosons) the appearance of AF long-range order is signaled by the Bose-Einstein condensation of the Schwinger bosons at zero temperature. In a three-dimensional system with an ordered ground state, thermally excited states reduce the spin correlations at finite temperatures. However, when the temperature is much higher than the typical coupling energy scale $J=4 t^{2} / U$, the spins are expected to be uncorrelated at large distances and the magnetization $m_{\mathrm{AF}}$ to vanish in the absence of an ordering field. This implies the existence of a phase transition at some temperature $T_{\mathrm{c}}$ between the ordered and disordered phases. According to our calculations, in the $U \rightarrow \infty$ localized limit $m_{\mathrm{AF}} \approx 0.422$, i.e. less than the mean field value $\Delta_{\mathrm{c}} / U=1 / 2$. Figure 1 shows the calculated antiferromagnetic phase diagram as a function of temperature and interaction strength. At strong coupling our theory clearly describes a Mott-Heisenberg antiferromagnet with an AF gap of order of $U$. As $U$ decreases, the Mott-Heisenberg antiferromagnet progressively evolves into a Slater antiferromagnet with an ex-potentially small AF gap. Also, in the weak interaction limit the $\mathrm{AF}$ order is destroyed at $U / t=0.676$ (see Fig. 1), due to the topological Berry phase term whose coefficient deviates from the localized spin value $S=1 / 2$ 


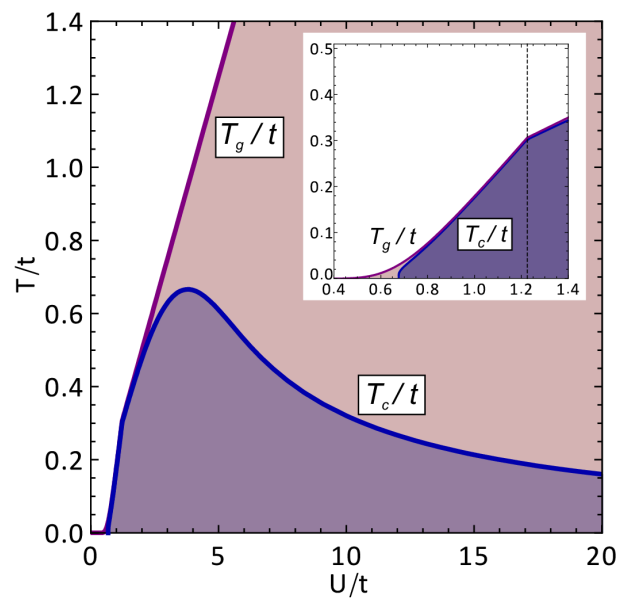

Fig. 1. The temperature-interaction phase diagram for the three-dimensional Hubbard model at half filling. Depicted is the temperature $T_{\mathrm{g}}$ (pink/light gray) for the vanishing of the gap parameter $\Delta_{\mathrm{c}}$ as well as the true critical temperature $T_{\mathrm{c}}$ (violet/dark gray) at which the log-range AF order ceases to exist, signalled by vanishing of $m_{\mathrm{AF}}$. The inset shows the details of the dependences in the weak coupling regime.

in the weak coupling limit $U / t$. The AF critical temperature has a maximum at $U / t \approx 3.78$. The comparison of our results with the previous work on the subject shows that numerical methods such as dynamical cluster approximation (DCA) [3] or dynamical mean-field theory approximation (DMFA) [4] give slightly higher values of $U / t \approx 7.5$, and $U / t \approx 10$, respectively. The methods based on a perturbation theory with respect to the interaction strength $[5,6]$ are unable to reproduce the maximum in the AF critical temperature as a function of $U / t$. The significantly higher values of $U / t$ resulting from DCA and DMFA have to be explained by the restricted ability of these methods while handling spatial fluctuations. Regarding the value of maximum of the critical temperature $T_{\mathrm{c}} / t \approx 0.667$ found here, it agrees with the result of Monte Carlo simulations by Scalettar et al. [7] $T_{\mathrm{c}} / t \approx 0.72$ and by Hirsch [8] who obtained $T_{\mathrm{c}} \approx W / 18 t$, where $W=12 t$ is the bandwidth for the $3 \mathrm{D}$ Hubbard model, i.e. $T_{\mathrm{c}} / t \approx 0.666$.

\section{Conclusions}

In conclusion, we have investigated antiferromagnetic phase diagram of the three-dimensional Hubbard model using $\mathrm{SU}(2) \times \mathrm{U}(1)$ rotating reference frame description. Calculations with the Hamiltonian for interacting electrons were reduced to calculation of functional integrals with a phase-angular action. Our implementation for the Hubbard model is consistent with the spin rotation symmetry and simultaneously is able to reproduce the Hartree-Fock result. Finally, we have compared the results of our calculations with a number of methods that were employed by other authors. 


\section{Acknowledgments}

One of us (T.K.K.) acknowledges the support by the Ministry of Education and Science (MEN) under grant No. 1P03B 10330 in the years 2006-2008. T.A.Z. wants to thank the Foundation for Polish Science for support within "Grants for Scholars" framework $(12 / 06)$.

\section{References}

[1] M.V. Berry, Proc. R. Soc. Lond. A 392, 451 (1984).

[2] H.J. Schulz, Phys. Rev. Lett. 65, 2462 (1990).

[3] P.R.C. Kent, M. Jarrell, T.A. Maier, Th. Pruschke, Phys. Rev. B 72, 060411(R) (2005).

[4] R. Staudt, M. Dzierzawa, A. Muramatsu, Eur. Phys. J. B 17, 411 (2000).

[5] P.G.J. van Dongen, Phys. Rev. Lett. 67, 757 (1991).

[6] A.N. Tahvildar-Zadeh, J.K. Freericks, M. Jarrell, Phys. Rev. B 55, 942 (1997).

[7] R.T. Scalettar, D.J. Scalapino, R.L. Sugar, D. Toussaint, Phys. Rev. B 39, 4711 (1989).

[8] J.E. Hirsch, Phys. Rev. B 35, 1851 (1987). 